Trauma Berufskrankh 2009 · 11 [Suppl 2]: 171-178

DOI 10.1007/s10039-008-1456-z

Online publiziert: 24. Dezember 2008

(c) Springer Medizin Verlag 2008

\author{
W. Strecker \\ Klinik für Orthopädie und Unfallchirurgie, Klinikum am Bruderwald, Bamberg
}

\title{
Kniegelenknahe Korrekturosteotomien
}

menten des Kniegelenks voraus, also im femoro-tibialen medialen und lateralen Kompartiment, ebenso patello-femoral. Daraus folgert zwangsläufig, dass jeder kniegelenknahen Korrekturosteotomie eine Arthroskopie vorgeschaltet werden muss [8]!

\section{Bildgebende Analyse der Beingeometrie}

Zur Beurteilung der lokalen Knochenqualität, von radiologischen Arthrosezeichen usw. sind lange Röntgenaufnahmen des betroffenen Kniegelenks ausreichend, ggf. ergänzt durch Patella-Tangential- oder Patella-Défilé-Aufnahmen in 30,60 und $90^{\circ}$.

Zur Beurteilung der frontalen Achsverhältnisse sind Ganzbeinaufnahmen im Stehen a.-p. zwingend zu fordern. Hierbei ist ein Beckengeradstand durch Unterfüttern des kürzeren Beins anzustreben. Projektionsfehler durch rotatorische Fehleinstellung sind auf jeden Fall zu vermeiden. Die sagittale Kniegelenkachse muss parallel zur Filmebene positioniert werden, was am besten mit einem parallelen Ausrichten der Kniekehle zur Filmebene zu erreichen ist. Deshalb ist zu fordern, dass der untersuchende bzw. der operierende Arzt den Patienten persönlich für die Ganzbeinaufnahme positioniert! Nur so lassen sich Projektionsfehler bei posttraumatischen oder kongenitalen Torsionsabweichungen, z. B. beim „inwardly-pointing-knee", vermeiden.

Im Fall von computertomographischen Längen- und Torsionswinkelmessungen sind Projektionsfehler ebenfalls zu vermeiden. Diese drohen insbesondere bei Streckdefiziten der Hüft- und Kniegelenke oder höhergradigen intraindividu- ellen Torsionsabweichungen von über $30^{\circ}$ [6]. In jedem Fall ist bei Streckdefiziten durch analoge Unterfütterung der gesunden Seite eine Parallelität von Ober- und Unterschenkel in der Sagittalebene zu bewerkstelligen. Die Ergebnisse der computertomographischen Analyse müssen von jedem Operateur eigenhändig überprüft werden. Sollte es zu Diskrepanzen zwischen bildgebender Analyse und klinischer Bewertung kommen, müssen derartige Widersprüche analysiert und aufgelöst werden, ggf. durch erneute Untersuchungen oder Einholung einer zweiten Meinung!

\section{Indikationen zur Korrekturosteotomie}

Folgende Einflussgrößen fließen in die letztliche Entscheidungsfindung mit ein:

- Biomechanik

- Funktion

- Kosmetik

- Lokale Situation

- Patient.

Es handelt sich in jedem Fall um eine abwägende Entscheidung einer individuellen Situation bei einem individuellen Patienten. Dessen Compliance und Grundstimmung müssen ebenso gewürdigt werden, wie etwaige systemische Pathologien und Risikofaktoren, wie Übergewicht, Diabetes mellitus, arterielle Verschlusskrankheit und Zigarettenrauchen. Aufgrund eigener Datenanalysen an mehr als 600 osteotomierten Patienten führen wir bei Rauchern keine aufklappenden oder distrahierenden Korrekturverfahren mehr durch. Mittlerweile bestehen keine Zweifel bezüglich der ungünstigen Aus- 
wirkungen von Zigarettenrauchen auf die Knochenheilung [3].

Die Bewertung der lokalen Gewebequalität beeinflusst entscheidend die Indikation zur Osteotomie und die Wahl der Osteotomiehöhe und -technik. Bei infektiösen oder postinfektiösen Zuständen an Knochen und Weichteilen ist bestenfalls in Ausnahmefällen eine lokale Operabilität gegeben.

An therapeutischen Zielsetzungen werden angestrebt:

- Schmerzreduktion

- Verbesserung der Funktion

- Vermeidung des Gelenkersatzes durch Harmonisierung der Belastungsverteilung der vorhandenen Knorpelreserven.

Unter Berücksichtigung dieser Kriterien werden in der Frontalebene bei kniegelenknahen Korrekturen eine mechanische Idealachse angestrebt, in der Sagittalebene eine volle Extension, in der Horizontalebene eine harmonische patello-femorale Kongruenz, Vermeidung von Längendifferenzen und ausgewogene, möglichst seitengleiche Torsionsverhältnisse mit jeweilig freiem rotatorischem Nulldurchgang im ipsilateralen Hüft- und Kniegelenk.

\section{Planerische Ist-Soll-Analyse}

\section{Frontale Achsabweichungen}

Die meisten Korrekturosteotomien an den unteren Extremitäten werden aufgrund von Deformitäten in der Frontalebene, also im Valgus- oder Varussinn, bei unikompartimenteller Gonarthrose indiziert. Auf der Grundlage von exakt projizierten Ganzbeinstandaufnahmen werden zunächst die femoralen und tibialen Kniebasiswinkel ausgemessen [14, 15]. Hierfür hat sich international die Nomenklatur von Dror Paley und John Herzensberg durchgesetzt [10]. Die planerische Ist-Soll-Analyse erfolgt in 6 Schritten:

Landmarken. Zunächst werden die anatomischen Landmarken eingezeichnet, also die Zentren von Hüftkopf, Kniegelenk und oberem Sprunggelenk. Die mechanische Tragachse nach Mikulicz [7] verbindet die Zentren von Hüft- und oberem Sprunggelenk und erlaubt somit Aussagen bezüglich einer Valgus- oder Varusdeformität.

\section{Anatomische Zuordnung der Defor-}

mität. Sie ist anhand der ermittelten Achs- und Gelenkwinkel möglich. Dazu werden zunächst der femorale Kniebasiswinkel, der mechanische laterale distale Femurwinkel (mLDFW; Normwert 85$90^{\circ}$ ), ermittelt, anschließend der mechanische (anatomische) mediale proximale Tibiawinkel (MPTW; Normwert $85-90^{\circ}$ ). Liegt einer dieser beiden gemessenen Kniebasiswinkel außerhalb der genannten Normen, muss die jeweilige Deformität dementsprechend femoral oder tibial angesiedelt sein. Zusätzlich ist der Kniespaltwinkel zu ermitteln, also der Winkel zwischen der femoralen und tibialen Kniebasislinie, als Ausdruck einer ligamentären Instabilität und des jeweiligen osteokartilaginären Substanzverlusts im Bereich des jeweils geschädigten femoro-tibialen Kompartiments.

Korrekturziel. Ist die Deformität tibial angesiedelt, kann die mechanische Tragachse antegrad, also von femoral her durch das Kniegelenk im erwünschten Belastungszentrum nach distal verlängert werden. Damit wird die angestrebte zukünftige Lage des Mittelpunkts des oberen Sprunggelenks (Soll) definiert.

Umgekehrt kann bei femoralen Deformitäten die mechanische Tragachse in retrograder Technik vom Unterschenkel, also von der Tibiadiaphyse ausgehend nach proximal verlängert werden. Das künftige Hüftkopfzentrum (Soll) lässt sich damit, unter Berücksichtigung entsprechender Längenkorrekturen, eindeutig festlegen. Hierbei schneidet die ideale mechanische Tragachse das Kniegelenk entsprechend der gewünschten femoro-tibialen Belastung medial vs. lateral in Abhängigkeit von der entsprechenden Knorpelqualität im medialen und lateralen Kompartiment. Die erwünschte Belastungsverteilung medial vs. lateral wird letztlich durch die arthroskopisch angetroffene Knorpelqualität definiert [8].

Höhe der Osteotomie. Femoral hat sich der metaphysäre Bereich, etwa 5-6 cm proximal der femoralen Kniebasislinie, als ideale Osteotomiehöhe bewährt. Die
Knochenheilung ist hier deutlich besser als in weiter proximal gelegenen Bereichen. Tibial entscheidet letztlich die patello-femorale Harmonie über die Osteotomiehöhe, sei es supra- oder infratuberositär. Grundsätzlich ist die supratuberositäre Osteotomiehöhe bezüglich der Knochenheilung günstiger.

Korrekturwinkel. Sind Osteotomiehöhe und Art der Osteotomietechnik, sei es als Keilosteotomie aufklappend oder zuklappend oder eine Form der längenneutralen Pendel- oder Domosteotomien, festgelegt, lässt sich der Korrekturwinkel ermitteln. Er liegt bei der antegraden Planung zwischen der Osteotomie sowie dem Ist- und Sollzentrum des oberen Sprunggelenks, analog dazu bei der retrograden Planung zwischen Osteotomiehöhe und dem Istund Sollzentrum des Hüftgelenks.

Bezüglich weiterer planerischer Einzelheiten wird auf eine detaillierte Übersichtsarbeit verwiesen [14].

\section{Sagittale Achsabweichungen}

Sie manifestieren sich klinisch als Beuge- oder Streckdefizit. Die bildgebende Analyse, am besten im Seitenvergleich, erfolgt durch lange seitliche Aufnahmen der Kniegelenke. Hier lassen sich Verkippungen der femoralen Kondylen und/ oder Veränderungen der physiologischen Retroversion des Tibiaplateaus verifizieren.

Der Ort der dominierenden Deformität beeinflusst auch hier die Höhe der Korrekturosteotomie, die femoral suprakondylär oder im Tibiakopfbereich infra- oder supratuberositär liegen kann. Sagittale Achsabweichungen werden ana$\log \mathrm{zu}$ den Korrekturen in der Frontalebene auf- oder zuklappend bzw. additiv oder subtraktiv extendiert oder flektiert. Bei eindimensionalen sagittalen Deformitäten sind auch alternative Verfahren der Achskorrektur denkbar, analog zur Dom-, Scheibenwischer- oder Pendelosteotomie in der Frontalebene.

\section{Translationsdeformitäten}

Ad-latus-Dislokationen begründen nur in sehr seltenen Fällen alleine eine Korrekturosteotomie. Meist bieten sich der- 
artige Maßnahmen im Rahmen von Korrekturen mehrdimensionaler Achsabweichungen an. Ein kniegelenknaher Achsenversatz ad latus kann sich ungünstig auf die patello-femorale Zentrierung auswirken und somit Ursache für eine patello-femorale Hyper- oder Hypokompression sein, sei es medial oder lateral.

Neben der grundlegenden klinischen Untersuchung geben Röntgen-Patella-Défilé-Aufnahmen bzw. eine computertomographische Abklärung über eine patellofemorale Dezentrierung Auskunft. Korrekturen werden im Allgemeinen durch quere Osteotomien femoral oder tibial durchgeführt.

\section{Torsionsabweichungen}

Relevante Torsionsabweichungen können zwanglos im Rahmen von queren Osteotomien, sei es femoral oder tibial, supraoder infratuberositär korrigiert werden. Voraussetzung ist eine entsprechende klinische und computertomographische/sonographische Analyse der Torsionsverhältnisse [15]. Von großer Bedeutung ist die Berücksichtigung der patello-femoralen Balance hinsichtlich geplanter Torsionskorrekturen. Lediglich infratuberositäre tibiale Torsionskorrekturen beeinflussen diese patello-femorale Relation nicht.

\section{Längendifferenzen}

Einzeitige Längenkorrekturen bis zu $3 \mathrm{~cm}$ am Oberschenkel [18] sind möglich, nicht jedoch am Unterschenkel. Zur Korrektur von längerstreckigen Unterschieden empfehlen sich kontinuierliche Verfahren der Kallusdistraktion, sei es intern mit Teleskopnägeln [1], kombiniert durch Marknägel mit unilateralen Distraktionsfixateuren [11] oder durch externe Distraktionsverfahren, wie unilaterale Distraktionssysteme oder Ringfixateure nach Ilisarov [5].

Grundsätzlich gilt, dass akute Längenänderungen nur dann erlaubt sind, wenn dies ohne Risiko von Dehnungsschäden oder Kinking zu bewerkstelligen ist. Bei potenziellen Dehnungsschäden sind immer kontinuierliche Korrekturverfahren zu bevorzugen.

Besonders kritisch zu bewerten sind Akutkorrekturen am proximalen Unter- schenkel. Akute Verlängerungen, Innentorsionen und Varisationen sind aus den genannten Gründen kontraindiziert!

\section{Kniegelenknahe Korrekturosteotomien}

Die einzeitigen Osteotomien im kniegelenknahen Bereich werden überwiegend als quere Osteotomie mit der oszillierenden Säge durchgeführt. Bei kontinuierlichen Korrekturverfahren, besonders femoral, sind Bohrloch- und Meißelosteoklasien im Einzelfall eine gute Alternative. Die diversen Techniken der Knochendurchtrennung sowie die Grundprinzipien von akuten und kontinuierlichen Korrekturen wurden bereits ausführlich dargestellt [16].

In Abhängigkeit von den Längenverhältnissen von Ober- und Unterschenkel im Seitenvergleich ist zu entscheiden, ob auf- oder zuklappende Verfahren zu bevorzugen sind. Die zuklappenden bzw. subtraktiven Keilosteotomien sind im Wesentlichen längenneutral, die aufklappenden Verfahren gehen meist mit einer Verlängerung des korrigierten Segments und des betroffenen Beins einher.

\section{Femorale Korrekturen}

\section{Einzeitige Osteotomieverfahren}

Hier haben sich längenneutrale suprakondyläre subtraktive Keilosteotomien bewährt. Die entsprechende Osteosynthese erfolgt von lateral bevorzugt mit einer $95^{\circ}$ Kondylenplatte, medial mit einer Rechtwinkelplatte. Während sich bei uns bislang laterale winkelstabile Plattensysteme aus Titan nicht bewährt haben, möglicherweise aufgrund zu hoher Weichteilfriktionen, scheinen diese medial günstiger $\mathrm{zu}$ sein und stellen mittlerweile eine Alternative zur Rechtwinkelplatte dar.

Bei Anwendung der genannten Klingenplattensysteme müssen die Plattenund insbesondere die Klingenlage auf der Planungszeichnung mit hoher Präzision festgelegt werden. Diese Genauigkeit ist auch bei der Umsetzung während der Operation zu fordern, insgesamt handelt es sich um eine durchaus anspruchsvolle Technik. Die durchlaufenen Operationsschritte werden im Folgenden besprochen:
Trauma Berufskrankh 2009 · 11 [Suppl 2]:

171-178

DOI 10.1007/s10039-008-1456-z

(C) Springer Medizin Verlag 2008

\section{W. Strecker \\ Kniegelenknahe Korrekturosteotomien}

\section{Zusammenfassung}

Voraussetzung für jede Korrektur kniegelenknaher Deformitäten ist eine umfassende

Analyse der knöchernen Geometrie, der Gelenkfunktion und -stabilität sowie der jeweiligen Weichteil- und Knochensituation. Die Planung der Deformitätenkorrektur schließt bereits die Entscheidung bezüglich des Orts der Korrektur, sei er femoral suprakondylär oder am Tibiakopf, der Art und der Technik der Knochendurchtrennung und der nachfolgenden knöchernen Rekonstruktion ein. Die gängigsten Korrekturverfahren femoral und tibial werden vorgestellt, ebenso wie bewährte Planungs- und Osteotomietechniken.

\section{Schlüsselwörter}

Knie · Deformität · Arthrose - Osteotomie . Korrektur

\section{Correction osteotomies around the knee}

\section{Abstract}

The analysis of leg deformities around the knee joint is based on a standardized clinical examination and on long-leg standing anteroposterior radiographs, which may be complemented by spot-film radiographs. Detailed planning is mandatory and includes decisions about the osteotomy level, angle of correction, osteotomy technique, and type of internal or external fixation. In this article, the most common correction techniques around the knee are presented.

\section{Keywords}

Knee - Deformity - Osteoarthritis . Osteotomy · Correction 


\section{Knieverletzungen}

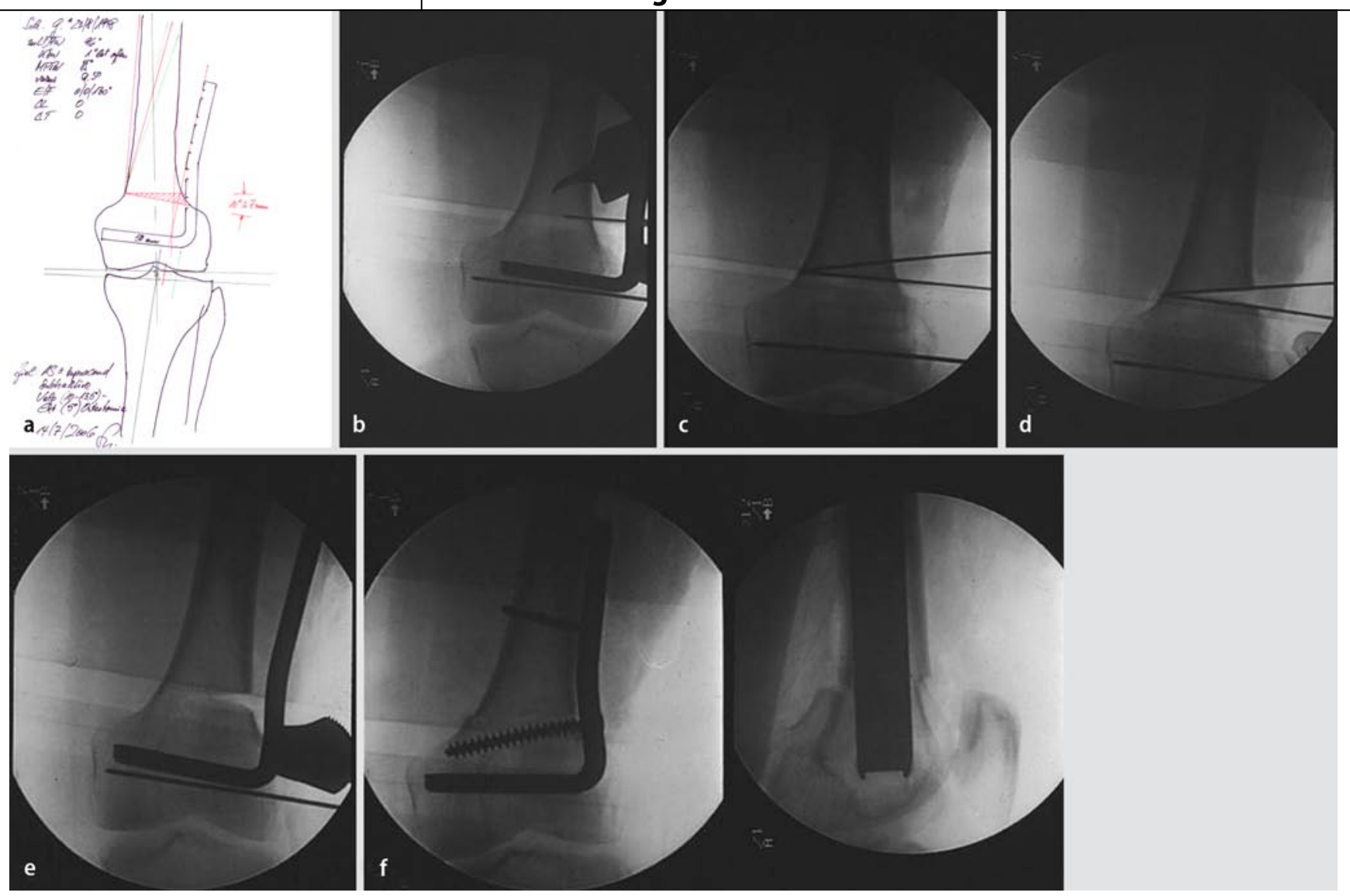

Abb. 1 A Fall 1, Genu varum, a planerische Analyse der Deformität, b Vorbereiten des Klingensitzes und Einbringen des Kirschner-Drahts, $\mathbf{c}$ Eindrehen des 2. suprakondylären Kirschner-Drahts, d Entfernung des Knochenkeils und Schwächen der medialen Kortikalis, e Eindrücken der $95^{\circ}$-Kondylenplatte, $\mathbf{f}$ kontinuierliches Schließen der zuklappenden Osteotomie, weitere Erläuterungen s. Text

Arthroskopie. Die Indikation zur Korrekturosteotomie wird noch einmal überprüft. Die Beurteilung der Knorpelqualität femoro-tibial medial vs. lateral bestimmt letztlich das definitive Ausmaß der Korrektur in der Frontalebene. Ansonsten erfolgen entsprechende therapeutische Maßnahmen an den Menisken, am Knorpel, wie etwa Mikrofrakturierung, ggf. auch Mosaikplastiken. Segensreich erscheint uns auch eine intraartikuläre Verbesserung von Streckdefiziten, etwa durch Notch-Plastik oder Resektion von Eminentiaosteophyten. Vor geplanten Valgisationen empfiehlt sich eine Resektion der Plica parapatellaris medialis.

Vorbereitung des Klingenlagers. Nach Beendigung der Arthroskopie werden der entsprechende operative Zugang suprakondylär lateral bzw. medial angelegt und die distale Femurdiaphyse und der Kondylenblock dargestellt. Unter Bildwandlerkontrolle in beiden Ebenen wird das Klingenlager analog zur Planungsskizze vorbereitet (Fall 1, $\bullet$ Abb. 1a,b).

Schanz-Schrauben als Zeiger. Sind Torsionskorrekturen vorgesehen, kann eine adäquate Torsionskontrolle durch 5-mmSchanz-Schrauben gewährleistet werden, die distal und proximal der geplanten Osteotomie außerhalb des Plattenlagers als Zeiger für die Torsion eingedreht werden.

Osteotomie. Kirschner-Drähte, deren Lage mittels Bildverstärker kontrolliert wird, dienen als Leitschiene für das Sägeblatt. Die Osteotomie selbst erfolgt unter dem Schutz von Hohmann-Hebeln mit der oszillierenden Säge. Hierbei ist ein ausreichend breites und nicht zu starkes Sägeblatt zu bevorzugen. Bei Keilosteotomien lassen sich durch entsprechende Neigung der Sägeblätter in der Sagittalebene evtl. erwünschte Extensions- oder Flexionskorrekturen realisieren. Bei Keil- osteotomien ohne gleichzeitige Torsionskorrektur sollte die gegenüberliegende Kortikalis nicht durchtrennt werden. Allerdings erscheint eine Schwächung der Kortikalis durch multiple Bohrungen etwa mit dem 2,0-mm-Bohrer vorteilhaft.

Einbringen der Platte. Die Klingenplatte wird möglichst sanft in das vorbereitete Klingenlager eingedrückt. Nach ihrer definitiven Positionierung wird die Osteotomie sukzessive geschlossen, wobei u. E. auf einen Plattenspanner verzichtet werden kann. Raum sparender ist eine schräg verlaufende „Spielschraube“ im proximalen Plattenloch, die ein kontinuierliches Schließen der Osteotomie mit Heranführen der Platte an die diaphysäre Femurkortikalis erlaubt.

Achskorrektur. Bei subtraktiven Keilosteotomien wird nach Schließen der Osteotomie ein flächiger Kontakt erreicht. Bei patello-femoraler Hyperkompression 

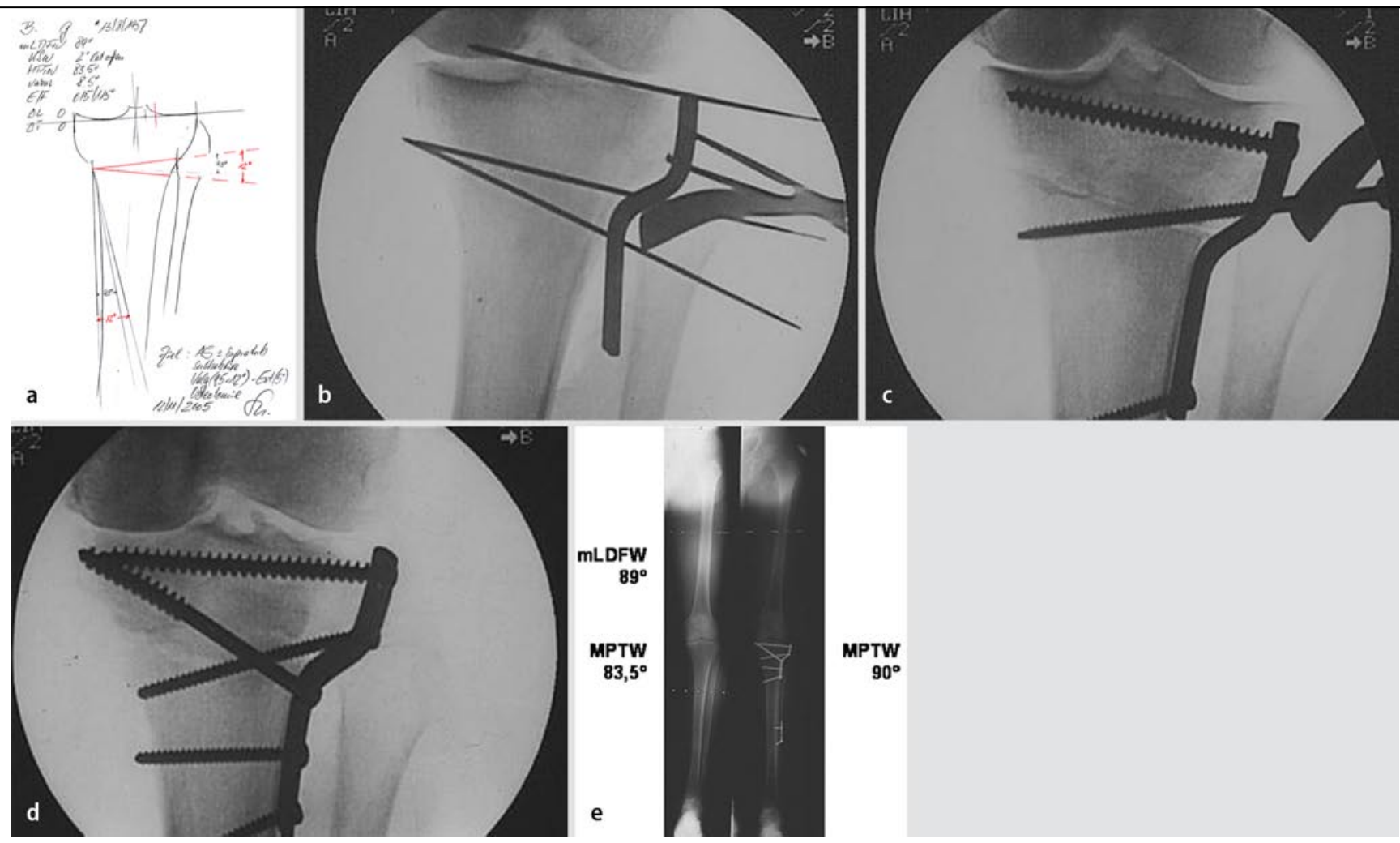

Abb. 2 \ Fall 2, 48-jähriger Patient mit Varusgonarthrose beidseits, a Analyse der Beingeometrie und Operationsplanung, b Einbringen der Kirschner-Drähte, c definitives Anlegen und Fixieren der Platte, Schließen der Osteotomie, Einbringen von 2 interfragmentären Zugschrauben, d Komplettieren der Osteosynthese, e zusätzliche Fibulaosteotomie, weitere Erläuterungen s. Text

erscheint uns eine milde Ventralisierung der Femurdiaphyse gegenüber dem Kondylenblock in einer Größenordnung von 2-3 mm erstrebenswert.

Osteosynthese. Sie wird bevorzugt mit einer interfragmentären Zugschraube komplettiert und abschließend mittels Bildverstärker in beiden Ebenen kontrolliert (Fall 1, $\mathbf{D}$ Abb. 1f).

\section{Kontinuierliche Korrekturverfahren}

Sie können am distalen Femur insbesondere bei posttraumatischen Zuständen mit wesentlichen Längendifferenzen angezeigt sein. In Abhängigkeit von den Knochen- und Weichteilverhältnissen können hierbei differenzialtherapeutisch eine Korrektur mittels retrogradem Marknagel in Kombination mit einem unilateralen Distraktionsfixateur erwogen werden oder alternativ ein rein externes Distraktionsverfahren mittels unilateralem Fixateur oder Ringfixateur.

Die Korrekturtechnik mit retrogradem Marknagel setzt ebenfalls eine pedantisch genaue Korrekturplanung voraus. Dies betrifft insbesondere den interkondylären Eintrittspunkt des Marknagels und die vorgegebene Richtung des Zieldrahts in der Frontal- und Sagittalebene. Planerische und operationstechnische Einzelheiten sind in der Literatur ausführlich beschrieben [13]. Das Korrekturverfahren mittels retrogradem Marknagel bietet Vorteile bezüglich der verschiedenen Korrekturoptionen bei insgesamt niedrigem Weichteiltrauma und erlaubt im Einzelfall u. U. eine Korrektur von Ober- und Unterschenkeldeformitäten über einen operativen Zugang. Besonders günstig erscheint diese Technik bei zu erwartender späterer Kniealloarthroplastik.

Gegenüber einem rein externen Korrekturverfahren kann die Tragezeit des Fixateur externe bei der kombinierten Technik deutlich verkürzt werden. Dies ist zum einen angenehmer für den Patienten und ermöglicht zum anderen meist eine bessere Kniegelenkfunktion aufgrund der zeitlich kürzeren Transfixierung der Weichteile durch die Schanz-Schrauben.

Während sich bei kongenitalen und weniger komplexen Deformitäten die
Winkelplattensysteme oder auch winkelstabilen Platten bewährt haben, stellt bei komplexen und insbesondere posttraumatischen Deformitäten die Kombination von retrogradem Marknagel mit unilateralen Distraktionsfixateuren eine interessante Alternative dar.

\section{Tibiale Korrekturen}

Die Indikation zur Korrekturosteotomie am Tibiakopf wird letztlich durch die bildgebende Analyse der Beingeometrie bestimmt: Während der mLDFW im Normbereich liegt, zeigt der MPTW entsprechende Abweichungen von der Norm von $85-90^{\circ}$. Die Osteotomiehöhe, sei es supra- oder infratuberositär, wird durch die Höhe der Patella beeinflusst, ebenso durch die patello-femorale Balance.

Ebenso wie bei suprakondylären Korrekturen bestimmen die Längenverhältnisse die Entscheidung über ein auf- oder zuklappendes Osteotomieverfahren. Ein diesbezüglich wichtiges Kriterium liefert auch die Stabilität der Kollateralbänder. Das Ausmaß einer sagittalen Korrektur 

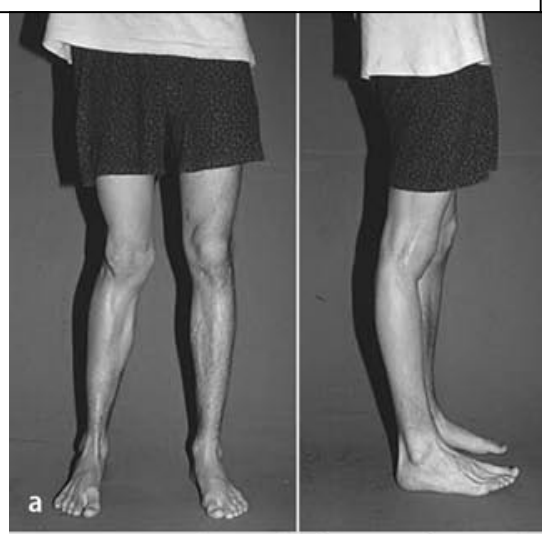

\section{Knieverletzungen}
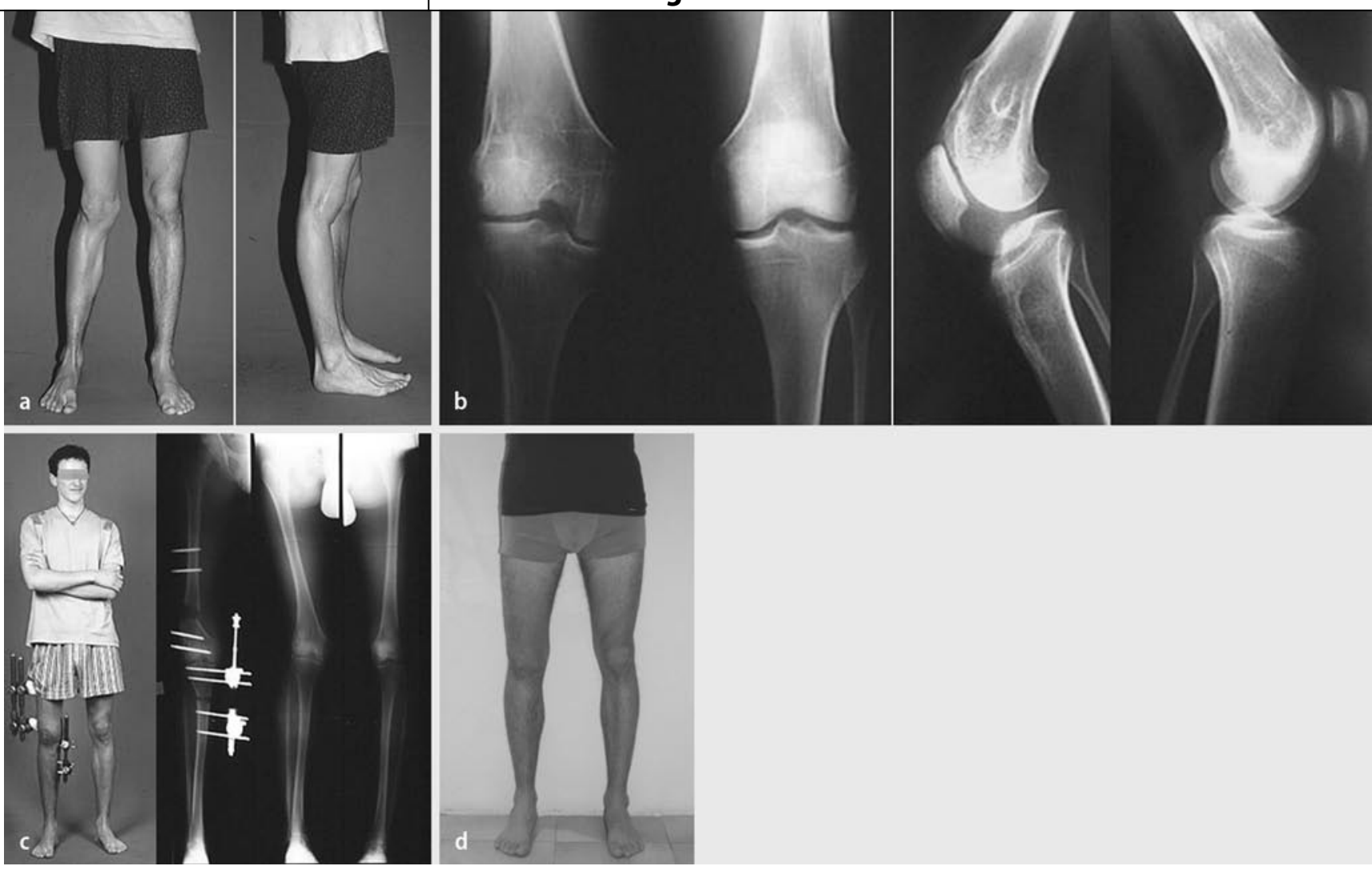

Abb. 3 A Fall 3, Patient, 20 Jahre, mit Komplexdeformität der Knieregion rechts, a klinische Untersuchung, b bildgebende Analyse, c klinisches Bild und Röntgenganzbeinaufnahme nach abgeschlossener Kallusdistraktion, d klinisches Ergebnis 2 Jahre nach dem Eingriff, weitere Erläuterungen s. Text

wird durch die Kniefunktion beeinflusst. In jedem Fall sind Streckdefizite zu beseitigen. Durch Modifikationen der tibialen Retroversion lassen sich Insuffizienzen des vorderen und hinteren Kreuzbandes in gewissem Maß positiv beeinflussen.

\section{Einzeitige Korrektur}

Bei der häufigen Varusgonarthrose konkurrieren im Wesentlichen 2 einzeitige Korrekturprinzipien.

\section{Lateral zuklappende, also subtraktive Valgisationsosteotomie. Sie findet be-} vorzugt supratuberositär mit nachfolgender Stabilisierung durch eine laterale Plattenosteosynthese Anwendung (Fall 2, - Abb. 2a-d). Die subtraktive Valgisationsosteotomie ist längenneutral. Korrekturen in der Sagittalebene und Torsionskorrekturen sind gleichzeitig möglich. Bei Valgisationen von mehr als $10^{\circ}$ ist allerdings eine zusätzliche Fibulaosteotomie nötig, die sich am Übergang vom mittleren zum distalen Drittel in Form einer Schrägosteotomie unschwer realisieren lässt (Fall 2, $\bullet$ Abb. 2e).
Medial aufklappende Osteotomie. Die Stabilisierung erfolgt durch eine winkelstabile Platte, etwa vom Typ Tomofix ${ }^{\mathrm{mi}}$.

Bei dieser Methode kommt es zu einer Unterschenkelverlängerung in einer Größenordnung von o,4-o,8 cm. Das ursprünglich vorgegebene supratuberositäre Verfahren geht mit einer Distalisierung der Patella einher. Daher empfiehlt sich bei Patella baja auf jeden Fall eine infratuberositäre biplanare Osteotomie. Gleichzeitige Torsionskorrekturen sind nicht möglich, nachteilig sind darüber hinaus relativ hohe Implantatkosten. Eine Fibulaosteotomie ist nicht erforderlich.

Die einzelnen Operationsschritte folgen im Wesentlichen dem oben dargestellten Vorgehen am suprakondylären Femur. Bezüglich der Einzelheiten der aufklappenden supratuberositären Tibiakopfvalgisationsosteotomie wird auf die einschlägige Literatur verwiesen [2]. Nachzutragen ist, dass wir aufklappende Korrekturverfahren beim Raucher aufgrund der kompromittierten Knochenheilung nicht anwenden [3]. Ebenfalls wichtig scheint uns die Empfehlung, nach aufklappenden
Korrekturverfahren die Metallentfernung aufgrund drohender sekundärer Korrekturverluste nicht vor Ablauf von 2 Jahren durchzuführen.

\section{Kontinuierliche Korrekturosteotomien}

Sie sind immer dann angezeigt, wenn einzeitige Korrekturverfahren an ihre Grenzen stoßen. Dies ist, wie bereits erwähnt, immer dann der Fall, wenn Dehnungsschäden neurovaskulärer Strukturen oder der Muskulatur drohen. Ebenfalls empfehlen sich kontinuierliche Korrekturverfahren bei Deformitäten in der Frontalund/oder Sagittalebene von über $20^{\circ}$.

Das Zusammentreffen von ipsilateralen femoralen und tibialen Deformitäten ist relativ selten, stellt dann aber besondere Anforderungen an die Analyse der Beingeometrie, die Planung der bifokalen Korrektur und deren Umsetzung. Vor einzeitigen femoralen und tibialen Korrekturen ist zu warnen! Hier empfehlen sich kontinuierliche Korrekturtechniken oder aber kombinierte Techniken mit einzeitiger Korrektur femoral und kontinuierlicher 
Korrektur tibial oder umgekehrt (Fall 3, - Abb. 3).

Vielleicht bringt die Zukunft eine ausreichend präzise intraoperative Korrekturkontrolle, etwa durch Anwendung der Navigation. Bis jetzt hat der Einsatz der Navigationstechnik bei komplexeren höherdimensionalen oder multifokalen Korrekturen keinen klinischen Stellenwert.

\section{Achskorrekturen mit gleichzeitigem Gelenkersatz}

Die Koinzidenz von fortgeschrittener Gonarthrose und höhergradiger, meist posttraumatischer Deformität ist selten, erfordert dann allerdings besondere Lösungsansätze. Diesbezüglich wird auf entsprechende Literaturbeiträge verwie$\operatorname{sen}[16]$.

\section{Kasuistik}

\section{Fall 1}

Bei der 55-jährigen Patientin mit Genu varum ergab die planerische Analyse der Deformität einen mLDFW von $96^{\circ}$ (Normwert: $85-90^{\circ}$ ), einen Kniespaltwinkel von $1^{\circ}$ sowie einen noch physiologischen MPTW von $85^{\circ}$ (• Abb. 1a). Es resultierte eine mechanische femoro-tibiale Varusdeformität von $9,5^{\circ}$, aufgrund deren femoraler Lokalisation sich eine suprakondyläre Valgisationsosteotomie empfahl. Es bestanden keine sagittale Deformität, keine Längen- und Torsionsabweichungen. Der Schnittpunkt der mechanischen Beinachse im lateralen femoro-tibialen Kompartiment sollte in Abhängigkeit von der arthroskopisch zu verifizierenden Knorpelqualität in einer GröBenordnung von $10-13,5^{\circ}$ liegen. In der Planungszeichnung wurden nicht nur der Korrekturwinkel, sondern auch die Implantatlage festgelegt.

Die Vorbereitung des Klingensitzes analog zur Planungszeichnung erfolgte nach den bekannten Operationsschritten [9]. Nach Einbringen des KirschnerDrahts für die geplante suprakondyläre Osteotomie etwa $5,5 \mathrm{~cm}$ proximal der femoralen Kniebasislinie ( $\mathbf{D}$ Abb. 1b) wurden der 2. suprakondyläre KirschnerDraht in dem nach lateral offenen Korrekturwinkel von hier $10^{\circ}$ für die subtraktive zuklappende Keilosteotomie eingedreht (- Abb. 1c), der Knochenkeil entfernt und die mediale Kortikalis mit einem 2,0mm-Bohrer geschwächt (• Abb. 1d). Beim Eindrücken der $95^{\circ}$-Kondylenplatte muss der Winkel der subtraktiven Keilosteotomie mit demjenigen zwischen lateraler Femurkortikalis und Platte übereinstimmen (• Abb. 1e). Die zuklappende Osteotomie wurde durch Einsetzen einer proximalen schrägen „Spielschraube“, die eine flächige Kompression im Bereich der Osteotomie gewährleistet, kontinuierlich geschlossen (- Abb. 1f), und die Osteosynthese wurde in typischer Weise vervollständigt. In der seitlichen Projektion stellte sich die erwünschte Ventralisierung der Diaphyse gegenüber dem Kondylenblock, die den patello-femoralen Anpressdruck vermindert, in einer Größenordnung von etwa $3 \mathrm{~mm}$ dar.

\section{Fall 2}

Die Analyse der Beingeometrie bei dem 48-jährigen Patienten mit beidseitiger Varusgonarthrose ergab einen mLDFW von $89^{\circ}$, einen Kniespaltwinkel von $2^{\circ}$, lateral offen, und einen MPTW von $83,5^{\circ}$, also unter der Norm von $85-90^{\circ}$ liegend (- Abb. 2a). Es resultierte ein mechanischer femoro-tibialer Varus von $8,5^{\circ}$.

Bei seitengleichen Längen- und Torsionsverhältnissen wurde eine supratuberositäre subtraktive Tibiakopfvalgisationsosteotomie von $9,5-12^{\circ}$ angestrebt, analog der Planungszeichnung. Das Streckdefizit von $5^{\circ}$ war ebenfalls korrekturbedürftig. Arthroskopisch zeigten sich eine Chondromalazie IV femoro-tibial medial und eine Chondromalazie I/II femoro-tibial lateral. Daraus leitete sich ein Korrekturwinkel von $12^{\circ}$ Valgus ab [8]. Analog der Planungsskizze wurden 2 KirschnerDrähte in einem nach lateral offenen Korrekturwinkel von $12^{\circ}$ eingebracht und eine 5-Loch-Großfragment-DCP aus Stahl anmodelliert. Hierbei musste der Winkel zwischen lateraler Tibiakortikalis und dem distalen Plattenanteil ebenfalls $12^{\circ}$ betragen (- Abb. 2b). Das Streckdefizit wurde arthroskopisch durch Notch-Plastik beseitigt und musste somit bei der Osteotomie nicht mehr berücksichtigt werden. Nach Schwächung der medialen Tibiakortikalis mit Bohrungen wurde die
Platte definitiv angelegt und mit einer Spongiosaschraube mit durchgehendem Gewinde im ersten Plattenloch fixiert. Die Osteotomie wurde mit einer distalen, schräg eingebrachten Kortikalisschraube allmählich geschlossen, bis eine flächige Kompression erreicht war. Anschließend wurden 2 interfragmentäre Zugschrauben eingebracht (- Abb. 2c). Beim Komplettieren der Osteotomie (- Abb. 2d) können sich die interfragmentären Schrauben durchbiegen, deshalb empfiehlt sich unbedingt eine Stahllegierung als Osteosynthesematerial, Titan ist diesen Biegekräften u. U. nicht gewachsen. Bei Korrekturwinkeln $>10^{\circ}$ Valgus wird eine zusätzliche Fibulaosteotomie im mittleren Drittel oder am Übergang vom mittleren zum distalen Drittel erforderlich, die mit einer kurzen Drittelrohrplatte stabilisiert werden kann (• Abb. 2e).

\section{Fall 3}

Der 20-Jährige zeigte eine Komplexdeformität der Knieregion rechts bei $\mathrm{Zu}$ stand nach mutmaßlich epiphysärer Kondylenverletzung und zahlreichen $\mathrm{He}$ miepiphysiodesen bzw. Epiphysiodesen (• Abb. 3a). Die klinische Untersuchung ergab - auf das rechte Knie bezogen - folgende Deformität:

- Valgus $11^{\circ}$

- Hyperextension $20^{\circ}$

- Außentorsion $20^{\circ}$

- Verkürzung $2,5 \mathrm{~cm}$

Die bildgebende Analyse (• Abb. 3b) der Deformität demaskierte eine komplexe intra- und extraartikuläre Deformität, sowohl femoral als auch tibial. Die Abweichungen lagen femoral bei:

- $18^{\circ}$ Valgus,

- $5^{\circ}$ Flexion,

- $2,5 \mathrm{~cm}$ Verkürzung und

- $10^{\circ}$ Außentorsion,

tibial bei

- $7^{\circ}$ Varus,

- $15^{\circ}$ Extension,

- $0,8 \mathrm{~cm}$ Verkürzung und

- $10^{\circ}$ Außentorsion.

Aus technischen und anatomischen Gründen lässt sich eine intraartikuläre Deformität meist nicht im Apex der Fehlstel- 
lung korrigieren und erfordert daher eine extraartikuläre Osteotomie. Dies geht zwangsläufig mit einer Translation einher, die letztlich funktionell ohne Relevanz ist. Entscheidend ist die physiologische Ausrichtung von Hüft-, Knie- und oberem Sprunggelenk zueinander, alle räumlichen Dimensionen betreffend. Derartige Komplexdeformitäten lassen sich in den meisten Fällen nur kontinuierlich korrigieren, im dargestellten Fall durch eine suprakondyläre Varisations-Extensions-Innentorsions-Osteoklasie femoral und tibial durch eine infratuberositäre Valgisations-Flexions-Innentorsions-Osteoklasie mit nachfolgender Verlängerung durch Kallusdistraktion im gewünschten Ausmaß. Das in - Abb. 3c dargestellte postoperative klinische Bild und die Röntgenganzbeinaufnahme zeigen die Situation nach abgeschlossener Kallusdistraktion. Die Distraktionsfixateure wurden nach adäquater Kallusreifung 4 Monate postoperativ entfernt.

Bei der klinischen und bildgebenden Analyse der postoperativen Beingeometrie 2 Jahre nach dem Eingriff fanden sich physiologische Verhältnisse in allen Dimensionen bei guter Funktion und Sportfähigkeit (• Abb. 3d).

\section{Korrespondenzadresse \\ Prof. Dr. W. Strecker}

Klinik für Orthopädie und Unfallchirurgie,

Klinikum am Bruderwald,

Buger Straße 80, 96049 Bamberg

chirurgie2@sozialstiftung-bamberg.de

Interessenkonflikt. Der korrespondierende Autor gibt an, dass kein Interessenkonflikt besteht.

\section{Literatur}

1. Baumgart R, Betz A, Schweiberer L (1997) A fully implantable motorized intramedullary nail for limb lengthening and bone transport. Clin Orthop 343:135-143

2. Galla M, Lobenhoffer P (2004) Die öffnende valgisierende Umstellungsosteotomie der proximalen Tibia mit dem TomoFix-Plattenfixateur. Operat Orthop Traumatol 16:397-417

3. Hoogendoorn JM, Simmermacher RKJ, Schellekens PPA, Van der Werken C (2002) Rauchen ist nachteilig für die Heilung von Knochen und Weichteilen. Unfallchirurg 105:76-81

4. Hsu RW, Himeno S, Coventry MB, Chao EY (1990) Normal axial alignment of the lower extremity and load bearing distribution at the knee. Clin Orthop 255:215-227

5. Ilizarov GA (1992) Transosseus synthesis. Springer, Heidelberg
6. Keppler P, Strecker W, Liebscher D, Kinzl L (1997) Projektionsfehler bei der computertomographischen Torsionswinkel- und Längenbestimmung an der unteren Extremität. In: Strecker W, Keppler P, Kinzl L (Hrsg) Posttraumatische Beindeformitäten - Analyse und Korrektur. Springer, Heidelberg, S 55-64

7. Mikulicz J (1878) Ueber individuelle Formdifferenzen am Femur und an der Tibia des Menschen. Arch Anat Physiol Anat Abt 1:351-404

8. Müller M, Strecker W (2008) Arthroscopy prior to osteotomy around the knee? Arch Orthop Trauma Surg 128:1217-1221

9. Müller ME, Allgöwer M, Schneider R, Willenegger H (1977) Manual der Osteosynthese, 2. Aufl. Springer, Heidelberg

10. Paley D (2002) Principles of deformity correction. Springer, Heidelberg

11. Paley D, Herzenberg JE, Paremain G, Bhave A (1997) Femoral lengthening over an intramedullary nail: a matched-case comparison with llisarov femoral lengthening. J Bone Joint Surg Am 79:1464-1480

12. Pandit $H$, Ward $T$, Hollinghurst $D$ et al (2005) Influence of surface geometry and the cam-post mechanism on the kinematics of total knee replacement. J Bone Joint Surg Br 87:940-945

13. Strecker W (2003) Korrekturosteotomie des distalen Femurs mit retrogradem Marknagel. Operat Orthop Traumatol 15:363-386

14. Strecker W (2006) Planerische Analyse kniegelenknaher Beinachsenabweichungen. Operat Orthop Traumatol 18:259-272

15. Strecker W, Keppler P (2002) Analyse und Korrektur von Beindeformitäten; Teil 1: Analyse. Unfallchirurg 73:811-829

16. Strecker W, Keppler P (2006) Therapie posttraumatischer Deformitäten. Trauma Berufskrankh [Suppl 3] 8:S317-S324

17. Strecker W, Keppler P, Gebhard F, Kinzl L (1997) Length and torsion of the lower limb. J Bone Joint Surg Br 79:1019-1023

18. Strecker W, Keppler P, Kinzl L (1999) Die treppenförmige subtrochantere Verlängerungsosteotomie des Oberschenkels. Operat Orthop Traumatol 11: $1-10$ 